\title{
Exact solution of a many body problem with nearest and next-nearest neighbour interactions
}

\author{
B. BASU-Mallick 由 And Anjan Kundu 色 \\ Theory Group, Saha Institute of Nuclear Physics, \\ 1/AF Bidhan Nagar, Calcutta 700 064, India.
}

\begin{abstract}
Recently a partially solvable many-body problem with nearest and next-nearest neighbour interactions is proposed [1]. We show that by adding a suitably chosen momentum dependent nearest neighbour interaction, such a model can be converted into an integrable system with Lax operator formulation and related conserved quantities. We also solve the eigenvalue problem for the model exactly and as a byproduct obtain some identities involving associated Laguerre polynomials.
\end{abstract}

It is well known that random matrix models have deep connection with some exactly solvable many-body systems having long-range interactions, namely Calogero [2] and Sutherland model [3]. However recently a new type of random matrix model, known as short-range Dyson model, has been introduced, where only the nearest neighbour energy levels interact with each other [4, 歌. For finding out the corresponding many-body system, Calogero and Sutherland like models with nearest and next-nearest neighbour interactions have been proposed recently [1]. Though many interesting results, e.g. construction of static correlation functions, partial solution of the energy eigenvalue problem, existence of off-diagonal long-range order etc. have been obtained, the integrability and complete solvability of these models could not be established. The Hamiltonian of such Calogero like model as considered in [1, 6] is given by

$H=-\frac{1}{2} \sum_{j=1}^{N} \frac{\partial^{2}}{\partial x_{j}^{2}}+\frac{\omega^{2}}{2} \sum_{j=1}^{N} x_{j}^{2}+\nu(\nu-1) \sum_{j=1}^{N-1} \frac{1}{\left(x_{j}-x_{j+1}\right)^{2}}-\nu^{2} \sum_{j=2}^{N-1} \frac{1}{\left(x_{j-1}-x_{j}\right)\left(x_{j}-x_{j+1}\right)}$.

Our aim here is to propose an integrable and completely solvable variant of the above model by suitably redefining its coupling constants and introducing an additional momentum dependent nearest neighbour interaction. The Hamiltonian of this model may

\footnotetext{
${ }^{1}$ email:biru@tnp.saha.ernet.in

2 email: anjan@tnp.saha.ernet.in
} 
be given as

$$
\begin{aligned}
H=-\frac{1}{2} \sum_{j=1}^{N} \frac{\partial^{2}}{\partial x_{j}^{2}} & +\frac{\omega^{2}}{2} \sum_{j=1}^{N} x_{j}^{2}+g_{1} \sum_{j=1}^{N-1} \frac{1}{\left(x_{j}-x_{j+1}\right)^{2}} \\
& +g_{2} \sum_{j=2}^{N-1} \frac{1}{\left(x_{j-1}-x_{j}\right)\left(x_{j}-x_{j+1}\right)}+g_{3} \sum_{j=1}^{N} f_{j} \frac{\partial}{\partial x_{j}},
\end{aligned}
$$

where the last term represents a momentum dependent interaction with

$$
f_{j}=\left(1-\delta_{1 j}\right) \frac{1}{x_{j}-x_{j-1}}+\left(1-\delta_{N, j}\right) \frac{1}{x_{j}-x_{j+1}}
$$

and the coupling constants are parametrised as

$$
g_{1}=\nu(i+\nu), \quad g_{2}=-\nu^{2}, \quad g_{3}=-i \nu
$$

It is easy to see that for real parameter $\nu$ the Hamiltonian (2) becomes hermitian. It has been shown recently [7] that the original Calogero model with long-range momentum dependent interaction retains its integrability and complete solvability. Similarly for the present model (2), involving only nearest neighbour momentum dependent interaction, one can establish the integrability and the existence of infinite number of conserved quantities. For this purpose one needs the associated Lax operator, which may be expressed as a $N \times N$ matrix with elements

$$
L_{j k}=\left(-i \frac{\partial}{\partial x_{j}}+\nu f_{j}\right) \delta_{j k} .
$$

One may check that this operator and Hamiltonian (2) satisfy the Lax equation

$$
\left[H, L^{ \pm}\right]= \pm \omega L^{ \pm}, \quad \text { where } L_{j k}^{ \pm}=L_{j k} \pm i \omega x_{j} \delta_{j k}
$$

Note that though the complementary Lax operator $M$ is absent in this Lax equation, it still leads to a set of conserved quantities given by $I_{m}=\sum_{j, k}\left(\left(L^{+} L^{-}\right)^{m}\right)_{j k}, m=1,2,3, \ldots$, ensuring the integrability of the system. Evidently Hamiltonian (2) is the first element of this set.

For solving the energy eigenvalue problem of model (1) and its other variants, the authors of [1, 6] have adopted two different approaches. The first one, as described in [8]-11], constructs the eigenfunctions by mapping the model to a system of free oscillators through a suitable similarity transformation. In the second approach, pioneered by Calogero [2], the solution for eigenfunctions is sought through an ansatz in the factorised form: $P_{k}(x) \Phi\left(r^{2}\right)$, where $P_{k}(x)$ is a homogeneous polynomial in all coordinates $x=x_{1}, x_{2}, \ldots, x_{N}$ and $\Phi\left(r^{2}\right)$ is a function of $r^{2}=\sum_{i=1}^{N} x_{i}^{2}$. Through both these methods some particular but exact eigenvalue solutions were obtained in [1, 6]. 
Due to the integrability property of our model (2), we hope to get the complete solution of its eigenvalue problem by adopting similar methods. Following the first approach, we find that there exists an unitary transformation which reduces the Hamiltonian (2) to a system of free oscillators:

$$
\left(\prod_{j=1}^{N-1}\left(x_{j}-x_{j+1}\right)\right)^{i \nu} H\left(\prod_{j=1}^{N-1}\left(x_{j}-x_{j+1}\right)\right)^{-i \nu}=H_{\text {free }}
$$

with

$$
H_{\text {free }}=-\frac{1}{2} \sum_{j=1}^{N} \frac{\partial^{2}}{\partial x_{j}^{2}}+\frac{\omega^{2}}{2} \sum_{j=1}^{N} x_{j}^{2} .
$$

As is well known, the eigenfunctions for the free oscillator model may be given by

$$
\phi_{n_{1}, n_{2}, \cdots, n_{N}}=e^{-\frac{\omega r^{2}}{2}} \prod_{j=1}^{N} H_{n_{j}}\left(\sqrt{\omega} x_{j}\right),
$$

where $r^{2}=\sum_{j=1}^{N} x_{j}^{2}$ and $H_{n}(x)$ is the Hermite polynomial of degree $n$. Therefore one can immediately write the eigenfunctions for our model (2) as

$$
\psi_{n_{1}, n_{2}, \cdots, n_{N}}=\left(\prod_{j=1}^{N-1}\left(x_{j}-x_{j+1}\right)\right)^{-i \nu} e^{-\frac{\omega r^{2}}{2}} \prod_{j=1}^{N} H_{n_{j}}\left(\sqrt{\omega} x_{j}\right) .
$$

The corresponding eigenvalues are evidently same as that of the free oscillators:

$$
E_{n_{1}, n_{2}, \cdots, n_{N}}=\omega\left(\frac{N}{2}+\sum_{j=1}^{N} n_{j}\right)
$$

The ground state wave function is clearly given by $\psi_{g r}=\left(\prod_{j=1}^{N-1}\left(x_{j}-x_{j+1}\right)\right)^{-i \nu} e^{-\frac{\omega r^{2}}{2}}$, with energy eigenvalue $E_{g r}=\frac{\omega N}{2}$. Thus we see that model (2), which is obtained from (11) by adding a momentum dependent term and redefining the coupling constants, becomes exactly solvable giving very simple spectrum. In a similar way, by adding suitably chosen momentum dependent interactions, the other variants of model (11) considered in [1], 6] can also be transformed to integrable systems and solved exactly.

We should note here that the unitary transformation (7), which brings our Hamiltonian to the free oscillator model, does not contain any singular terms. Consequently the eigenfunctions (10) form a complete set and the spectrum coincides with that of distinguishable oscillators. In contrast, the similarity transformations which map the standard Calogero model as well as (11) into the free oscillator model contain singular terms. In the case of Calogero model such singularities can be avoided by symmetrising the eigenfunctions of free oscillators in all coordinates [8]. As a result the eigenfunctions of Calogero model represent a complete set with the excitation spectrum becoming equivalent to that 
of bosonic oscillators. However in the case of (11) such singularities can not be removed in general even by taking symmetrised eigenfunctions and that makes the completeness of eigenfunctions difficult to establish.

It may be noted that both (11) and our model (2) reduce to the free oscillator model at $\nu \rightarrow 0$. Therefore it may be expected that a link should exist between the solutions of these two models at $\nu \rightarrow 0$ limit. To examine this possibility we follow the second approach, as mentioned above, for solving the model (2) and find that the corresponding eigenfunctions can be obtained in the factorised form

$$
\Phi_{n, k}=e^{-\frac{\omega r^{2}}{2}}\left(\prod_{j=1}^{N-1}\left(x_{j}-x_{j+1}\right)\right)^{-i \nu} L_{n}^{\frac{N}{2}+k-1}\left(\omega r^{2}\right) P_{k}(x) .
$$

Here $P_{k}(x)$ is a homogeneous polynomial of degree $k$ satisfying the equation

$$
\sum_{j=1}^{N} \frac{\partial^{2}}{\partial x_{j}^{2}} P_{k}\left(x_{1}, x_{2}, \cdots, x_{N}\right)=0
$$

and $L_{n}^{\frac{N}{2}+k-1}\left(\omega r^{2}\right)$ is the associated Laguerre polynomial. The corresponding eigenvalue would be $E_{n, k}=\omega\left(\frac{N}{2}+2 n+k\right)$. A class of trivial solutions of (13) with $k \leq N$ is clearly given by

$$
P_{k}(x)=x_{\alpha_{1}} x_{\alpha_{2}} \cdots x_{\alpha_{k}}
$$

where $\alpha_{l}$ s are all different integers ranging from 1 to $N$. For finding nontrivial solutions of (13) we express $P_{k}(x)$ in a general form

$$
P_{k}(x)=\sum_{r_{1}+r_{2}+\cdots+r_{N}=k} \frac{k !}{r_{1} ! r_{2} ! \cdots r_{k} !} f_{r_{1}, r_{2}, \cdots, r_{N}}^{k} x_{1}^{r_{1}} x_{2}^{r_{2}} \cdots x_{N}^{r_{N}},
$$

where $f_{r_{1}, r_{2}, \cdots, r_{N}}^{k}$ are yet unknown coefficients and the summation variables $r_{j}$ are nonnegative integers. The equation (13) however puts constraints on these coefficients as

$$
\sum_{j=1}^{N} f_{r_{1}, r_{2}, \cdots, r_{j-1}, r_{j}+2, r_{j+1}, r_{j+2}, \cdots, r_{N}}^{k}=0
$$

where $\left\{r_{j}\right\}$ represents any partition of $k-2$ satisfying $\sum_{j=1}^{N} r_{j}=k-2$. Such constraints are evidently different for different choices of $\left\{r_{j}\right\}$. However since the number of constraints is less than the number of unknown coefficients, they can not be fixed uniquely unless one imposes some extra relationship among them. Therefore the corresponding polynomial (15) may not be symmetric in all its coordinates in general. For example for $k=2$ one gets the solution

$$
P_{2}(x)=a_{1} x_{1}^{2}+a_{2} x_{2}^{2}+\cdots+a_{N} x_{N}^{2}, \quad \text { with } \sum_{j=1}^{N} a_{j}=0
$$


which can not be presented in a symmetric form. On the other hand for higher $k$ one may obtain symmetric polynomials as solutions of (16). For $k=3$ such a solution is explicitly given by

$$
P_{3}(x)=a \sum_{j=1}^{N} x_{j}^{3}+b \sum_{j \neq k} x_{j}^{2} x_{k},
$$

where $3 a+(N-1) b=0$. For $k=4$ we get however two independent solutions of the form

$$
P_{4}(x)=a \sum_{j=1}^{N} x_{j}^{4}+b \sum_{j \neq k} x_{j}^{2} x_{k}^{2}
$$

with the constraint $3 a+(N-1) b=0$, and

$$
P_{4}(x)=c \sum_{j \neq k} x_{j}^{3} x_{k}+d \sum_{j \neq k \neq l \neq j} x_{j}^{2} x_{k} x_{l},
$$

with $3 c+(N-2) d=0$.

We may compare now the above solutions at $\nu \rightarrow 0$ with those of (11), as obtained in [1], at the same limit. For the case $k=2$ while we get two solutions with $P_{2}(x)$ taking the trivial form (14) as well as the nontrivial one (17), [1] gives only one solution with trivial form of $P_{2}(x)$. For $k=3$ we get again two independent solutions corresponding to (18) and (14), while in [1] one finds only a particular combination of them. Similarly for $k=4$ we get three independent solutions in contrast to a single solution of [1]. This picture prevails also for higher $k$.

Finally we would like to comment on some useful identities, which may be derived by comparing the eigenfunctions (10) and (12) obtained through two different approaches. Expressing each of the solutions (12) as some linear combinations of the complete set (10), one would generate such an identity involving associated Laguerre and Hermite polynomials. By using this procedure along with the familiar conversion formulas like $H_{2 n}(x)=(-1)^{n} 2^{2 n} n ! L_{n}^{-\frac{1}{2}}\left(x^{2}\right), H_{2 n+1}(x)=(-1)^{n} 2^{2 n+1} n ! x L_{n}^{\frac{1}{2}}\left(x^{2}\right)$, we find interestingly that (12) with the trivial solution (14) for $P_{k}(x)$ leads to a well known summation formula [12] for associated Laguerre polynomials:

$$
L_{n}^{\frac{N}{2}+k-1}\left(\omega r^{2}\right)=\sum_{n_{1}+n_{2}+\cdots+n_{N}=n} \prod_{r=1}^{k} L_{n_{r}}^{\frac{1}{2}}\left(\omega x_{r}^{2}\right) \prod_{r=k+1}^{N} L_{n_{r}}^{-\frac{1}{2}}\left(\omega x_{r}^{2}\right) .
$$

On the other hand, (12) with nontrivial solutions for $P_{k}(x)$ leads to apparently new identities involving associated Laguerre polynomials. For example, the solution (17) for $N=2$ giving $P_{2}(x)=x_{1}^{2}-x_{2}^{2}$ results an identity of the form

$$
L_{n}^{2}\left(\omega x_{1}^{2}+\omega x_{2}^{2}\right)=\frac{1}{\omega\left(x_{1}^{2}-x_{2}^{2}\right)} \sum_{s=0}^{n+1}(2 s-n-1) L_{n-s+1}^{-\frac{1}{2}}\left(\omega x_{1}^{2}\right) L_{s}^{-\frac{1}{2}}\left(\omega x_{2}^{2}\right) .
$$

We hope to find similar identities for higher values of $k$ and $N$ and report elsewhere [13]. 


\section{References}

[1] S.R. Jain and A. Khare, Phys. Lett. A 262 (1999) 35;

G. Auberson, S.R. Jain and A. Khare, A class of N-body problems with nearest and next-to-nearest neighbour interactions, cond-mat/0004012.

[2] F. Calogero, J. Math. Phys. 10 (1969) 2191; 12 (1971) 419.

[3] B. Sutherland, J. Math. Phys. 12 (1971) 246, 251.

[4] B. Grémaud and S.R. Jain, J. Phys. A 31 (1998) L637.

[5] E. Bogomolny, U. Gerland and C. Schmit, Phys. Rev. E 59 (1999) R1315.

[6] M. Ezung, N. Gurappa, A. Khare and P.K. Panigrahi, Algebraic study of quantum many-body systems with nearest and next-to-nearest neighbour long-range interactions, cond-mat/0007005.

[7] B. Basu-Mallick and Anjan Kundu, Exact solution of Calogero model with competing long-range interactions, cond-mat/0003425, to appear in Phys. Rev. B.

[8] N. Gurappa and P.K. Panigrahi, Phys. Rev. B 59, R2490 (1999).

[9] K. Sogo, J. Phys. Soc. Jpn. 65, 3097 (1996).

[10] T.H. Baker and P.J. Forrester, Nucl. Phys. B 492, 682 (1997).

[11] H. Ujino, A. Nishino and M. Wadati, J. Phys. Soc. Jpn. 67, 2658 (1998).

[12] I.S. Gradshteyn and I.M. Ryzhik, Tables of Integrals, Series and Products (Acad. Press, 1965).

[13] B. Basu-Mallick and Anjan Kundu, Under preparation 